\title{
Generation of Nonclassical Motional States of a Trapped Atom
}

\author{
D. M. Meekhof, C. Monroe, B. E. King, W. M. Itano, and D. J. Wineland \\ Time and Frequency Division, National Institute of Standards and Technology, Boulder, Colorado 80303-3328
}

(Received 11 October 1995)

\begin{abstract}
We report the creation of thermal, Fock, coherent, and squeezed states of motion of a harmonically bound ${ }^{9} \mathrm{Be}^{+}$ion. The last three states are coherently prepared from an ion which has been initially laser cooled to the zero point of motion. The ion is trapped in the regime where the coupling between its motional and internal states, due to applied (classical) radiation, can be described by a JaynesCummings-type interaction. With this coupling, the evolution of the internal atomic state provides a signature of the number state distribution of the motion.
\end{abstract}

PACS numbers: 42.50.Vk, 32.80.Pj, 32.80.Qk

Nonclassical states of the harmonic oscillator associated with a single mode of the radiation field (for example, squeezed states) have been a subject of considerable interest. One method for analyzing these states has been through the dynamics of a single, two-level atom which radiatively couples to the single mode radiation field. This system, described by the Jaynes-Cummings model (JCM) interaction [1,2], is important to the field of cavity QED [3].

Nonclassical states of motion occur naturally on an atomic scale, for example, for electrons in atoms and atoms in molecules. On a macroscopic scale, the benefits of nonclassical mechanical states, such as squeezed states, for detection of gravitational waves have been appreciated for some time [4], but so far these states have not been realized. More recently, there has been interest in the generation and detection of nonclassical states of motion for an atom confined in a macroscopic, harmonic trap; for trapped ions, see Refs. [5-16]. These states are of interest from the standpoint of quantum measurement concepts and may facilitate other measurements such as sensitive detection [5,7,13] or quantum computation [17].

In this Letter we report the generation and detection of thermal, Fock, coherent, and squeezed states of motion of a single ${ }^{9} \mathrm{Be}^{+}$ion confined in an rf (Paul) trap. We detect the state of atomic motion by observing the evolution of the atom's internal levels [6,11] (e.g., collapse and revival) under the influence of a JCM-type interaction realized with the application of external (classical) fields. Under certain conditions, the interaction Hamiltonian is formally equivalent to the JCM Hamiltonian of cavity QED. Here, the harmonic motion of the atom replaces the single mode of the radiation field. The coupling can be realized by applying quasistatic fields [7], traveling-wave fields $[6,10,13,15]$, or standing-wave laser fields $[8,9,12]$. In each case the coupling $\mathcal{H}_{I}=-\boldsymbol{\mu} \cdot \mathbf{E}(\mathbf{r})$ between internal and motional states is induced by the atom's motion through the spatially inhomogeneous electromagnetic field $\mathbf{E}(\mathbf{r})$, where $\boldsymbol{\mu}$ is the atomic dipole operator.

In the present experiment, we drive stimulated Raman transitions between two hyperfine ground states by apply- ing a pair of traveling-wave laser beams detuned from an excited electronic state [18]. The resulting interaction between these internal states $|S\rangle$ (denoted $|\downarrow\rangle$ and $|\uparrow\rangle$ ) and motional harmonic oscillator states $|n\rangle$ and $\left|n^{\prime}\right\rangle$ in the $x$ direction is given by matrix elements

$$
\begin{aligned}
\left\langle S^{\prime}, n^{\prime}\left|\mathcal{H}_{I}\right| S, n\right\rangle= & \hbar \Omega\left\langle S^{\prime}, n^{\prime}\right| \sigma_{+} e^{i \eta\left(a+a^{\dagger}\right)} \\
& +\sigma_{-} e^{-i \eta\left(a+a^{\dagger}\right)}|S, n\rangle
\end{aligned}
$$

in a frame which rotates at the difference frequency of the laser beams. In this expression, $\sigma_{+}\left(\sigma_{-}\right)$is the raising (lowering) operator for the internal atomic state, $a^{\dagger}(a)$ is the harmonic oscillator raising (lowering) operator, and $\Omega$ is the Raman coupling parameter [5,13,18]. The LambDicke parameter is defined by $\eta \equiv \delta k x_{0}$, where $\delta k$ is the wave-vector difference of the two Raman beams along $x$, and $x_{0}=\sqrt{\hbar / 2 m \omega}$ is the spread of the $|n=0\rangle$ wave function in the harmonic well of frequency $\omega$.

The order $n^{\prime}-n$ of the vibrational coupling is selected by tuning the Raman beam difference frequency. For example, by tuning to the first red sideband in the Raman spectrum, we resonantly enhance the term which drives transitions between states $|\downarrow, n\rangle$ and $|\uparrow, n-1\rangle$. In the Lamb-Dicke limit $\left[\delta k \sqrt{\left\langle x^{2}\right\rangle} \ll 1, x=x_{0}\left(a+a^{\dagger}\right)\right]$, the exponentials in Eq. (1) can be expanded to lowest order, resulting in the operator $\eta\left(a \sigma_{+}+a^{\dagger} \boldsymbol{\sigma}_{-}\right)$, which corresponds to the usual JCM operator. We can easily control the strength and duration of the interaction by varying the intensity and time the lasers are applied. By choosing other laser tunings, we can select other operators such as the antiJCM operator $\eta\left(a^{\dagger} \sigma_{+}+a \sigma_{-}\right)$at the first blue sideband (which is not present in cavity QED) or the "two-phonon" JCM operator $\left(\eta^{2} / 2\right)\left(a^{2} \sigma_{+}+a^{\dagger 2} \sigma_{-}\right)$at the second red sideband. In this experiment, the higher-order terms in the expansion of the exponential in Eq. (1) must also be taken into account [19]. Reference [20] has explicitly discussed the consequences of these higher-order terms on the trapped ion internal and motional state dynamics.

Additional differences from cavity-QED experiments include the methods of state generation available (described below) and the relatively small decoherence. In all but the case of thermal states, we coherently prepare 
the states starting with an ion which has been laser cooled to the $|n=0\rangle$ ground state of motion [18]; this cooling is crucial to preserve the consistency of the generated states. Decoherence in these experiments is small enough that the atomic population coherence survives for many Rabi cycles of the JCM interaction - the "strong coupling" regime of cavity QED [3].

A single ${ }^{9} \mathrm{Be}^{+}$ion is stored in a strong rf Paul trap [21] with a pseudopotential oscillation frequency along $x$ of $\omega / 2 \pi \approx 11.2 \mathrm{MHz}$. The ion is cooled using sideband cooling with stimulated Raman transitions [18] between the ${ }^{2} S_{1 / 2}\left(F=2, m_{F}=2\right)$ and ${ }^{2} S_{1 / 2}\left(F=1, m_{F}=1\right)$ hyperfine ground states, which are denoted by $|\downarrow\rangle$ and $|\uparrow\rangle$, respectively, and are separated by $\approx 1.25 \mathrm{GHz}$. This prepares the ion in the $|S=\downarrow, n=0\rangle$ state more than $95 \%$ of the time. The Raman beam wave vectors point at $45^{\circ}$ to the $x$ axis with their wave-vector difference nearly along the $x$ axis of the trap [the Lamb-Dicke parameter is $\eta=0.202(5)]$, so the Raman transitions are highly insensitive to motion in the $y$ or $z$ directions. The beams are detuned $\approx 12 \mathrm{GHz}$ to the red of the ${ }^{2} P_{1 / 2}$ excited state with approximately $0.5 \mathrm{~mW}$ of power in each beam, so that the Raman transition coupling is $\Omega / 2 \pi \approx 500 \mathrm{kHz}$, and the vibrational structure is clearly resolved.

Once the ion is prepared in the $|\downarrow, 0\rangle$ state, we create the desired motional state as described below. We then acquire the signature of the motional state as follows. The Raman beams are tuned to the first blue sideband and applied for a time $t$. The population of the $|\downarrow\rangle$ level is then measured by applying $\sigma^{+}$-polarized radiation on the $|\downarrow\rangle \rightarrow{ }^{2} P_{3 / 2}$ cycling transition and detecting the fluorescence [18]. This experiment is repeated at a rate of a few kilohertz, while $t$ is slowly stepped, accumulating the probability $P_{\downarrow}(t)$ of occupation in $|\downarrow\rangle$.

Fock states of motion can in principle be produced by quantum jumps [9,16], adiabatic passage [12], or trapping states [14]; here we use an alternate technique. Since the ion is initially cooled to the $|\downarrow, 0\rangle$ Fock state, we create higher- $n$ Fock states by simply applying a sequence of Rabi $\pi$ pulses of laser radiation on the blue sideband, red sideband, or carrier. For example, the $|\uparrow, 2\rangle$ state is prepared by using blue sideband, red sideband, and carrier $\pi$ pulses in succession, so that the ion steps through the states $|\downarrow, 0\rangle,|\uparrow, 1\rangle,|\downarrow, 2\rangle$, and $|\uparrow, 2\rangle$.

We create a series of Fock states, $|\downarrow, n\rangle$, and record $P_{\downarrow}(t)$. The expected signal is $P_{\downarrow}(t)=\cos ^{2}\left(\Omega_{n, n+1} t\right) \exp \left(-\gamma_{n} t\right)$, where $\Omega_{n, n+1}$ is the Rabi frequency and $\gamma_{n}$ is the decoherence rate between levels $|n\rangle$ and $|n+1\rangle$. The measured $P_{\downarrow}(t)$ for an initial $|\downarrow, n=0\rangle$ Fock state is shown in Fig. 1(a) and fitted by this equation, yielding $\Omega_{0,1}=$ 94(1) $\mathrm{kHz}$ and $\gamma_{0}=11.9(4) \mathrm{kHz}$. Note that $\Omega_{0,1}$ is much greater than $\gamma_{0}$, satisfying the strong coupling condition. We believe the decoherence is due to technical problemsprimarily intensity fluctuations of the laser beams and instabilities of the trap drive frequency and voltage amplitude. The observed increase of $\gamma_{n}$ with $n$ [we find $\gamma_{n} \approx$
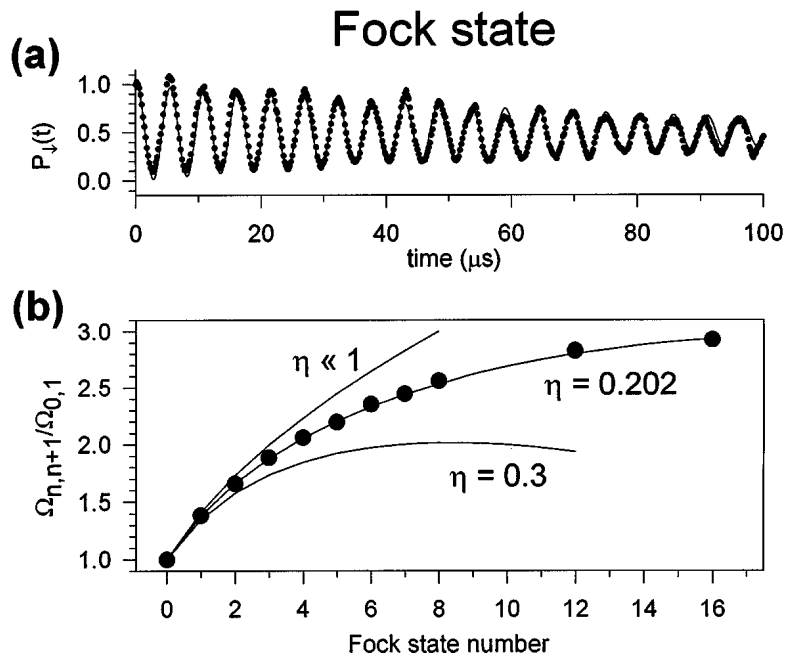

FIG. 1. (a) $P_{\downarrow}(t)$ for an initial $|\downarrow, 0\rangle$ Fock state driven by a JCM-type interaction provided by tuning the stimulated Raman beams to the first blue sideband. The solid line is a fit by an exponentially decaying sinusoid. (b) The relative Rabi frequencies $\Omega_{n, n+1} / \Omega_{0,1}$ vs the prepared Fock state number $n$. The lines represent the predictions of the nonlinear JCM for certain Lamb-Dicke parameters, showing very good agreement with the known Lamb-Dicke parameter $\eta=0.202(5)$. The $\eta \ll 1$ line corresponds to the LambDicke limit: $\Omega_{n, n+1} / \Omega_{0,1}=\sqrt{n+1}$.

$\left.\gamma_{0}(n+1)^{0.7}\right]$ is qualitatively consistent with this view. In the Lamb-Dicke limit the Rabi frequency between levels $|\downarrow, n\rangle$ and $|\uparrow, n+1\rangle$ is $\Omega_{n, n+1}=\sqrt{n+1} \eta \Omega$. If the Lamb-Dicke limit is not satisfied, nonlinear effects in the interaction modify these rates $[19,20]$. The measured Rabi frequency ratios $\Omega_{n, n+1} / \Omega_{0,1}$ are plotted in Fig. 1(b), showing very good agreement with the JCM for the trap's Lamb-Dicke parameter $\eta=0.202$.

When the ion's motion is not in a Fock state, $P_{\downarrow}(t)$ shows a more complicated structure. The motional state is characterized by a density operator whose diagonal elements have a number distribution $P_{n}$ leading to

$$
P_{\downarrow}(t)=\sum_{n=0} P_{n} \cos ^{2}\left(\Omega_{n, n+1} t\right) e^{-\gamma_{n} t} .
$$

For a thermal distribution $P_{n}=N[\bar{n} /(1+\bar{n})]^{n}$, where $N$ is a normalization constant and $\bar{n}$ is the average vibrational quantum number. By performing Doppler cooling on the $|\downarrow\rangle \rightarrow P_{3 / 2}$ cycling transition [18], we generate a thermal state of motion [22]. The value of $\bar{n}$ can be controlled by the Doppler detuning. An example of $P_{\downarrow}(t)$ data for a thermal state of motion is given in Fig. 2. To demonstrate consistency with a thermal state of motion, the timedomain data are fitted by Eq. (2) with a thermal population distribution for $P_{n}$. The signal scale and $\bar{n}$ are allowed to vary in the fit. Values for the base Rabi frequency $\Omega_{0,1}$ and base decay rate $\gamma_{0}$ (from which the other rates are scaled using the Fock state data) are obtained from a separate trace of $P_{\downarrow}(t)$ for an initial $|\downarrow, 0\rangle$ state, as in Fig. 1(a). For Fig. 2, the fit yields $\bar{n}=1.3 \pm 0.1$. The inset shows the results of 

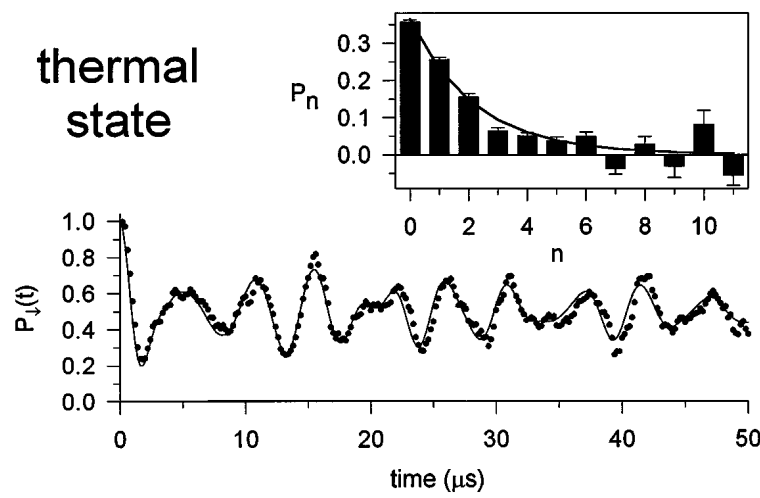

FIG. 2. $P_{\downarrow}(t)$ for a thermal state. The data (points) are fitted (line) by a superposition of Fock states with $P_{n}$ given by a thermal state distribution. The fit allows $\bar{n}$ to vary, finding $1.3 \pm 0.1$. The inset shows the decomposition of the data onto the Fock state components (bars) with a fit (line) by the expected exponential distribution, yielding $1.5 \pm 0.1$.

an independent analysis (the frequency-domain analysis). In this case, we directly extract the populations of the various $|n\rangle$ levels. Since the Fock state parameters $\Omega_{n, n+1}$ and $\gamma_{n}$ are well characterized, the time-domain data can be decomposed into Fock-state components. Equation (2) is linear in the $P_{n}$, so we use singular-value decomposition [23] to extract the probabilities, shown in the inset to Fig. 2. The probabilities are fitted by an exponential, yielding $\bar{n}=1.5 \pm 0.1$. Finally, we independently measure $\bar{n}$ by comparing the size of the red and blue sidebands [18], yielding $\bar{n}=1.5 \pm 0.2$.

A coherent state of motion of the ion corresponds to a displaced zero-point wave packet oscillating in the potential well. The distribution among Fock states is Poissonian, $P_{n}=\left(\bar{n}^{n} e^{-\bar{n}}\right) / n$ !. As predicted by the JCM, the internal-state evolution $P_{\downarrow}(t)$ will undergo quantum collapses and revivals [24]. These revivals are a purely quantum effect due to the discrete energy levels and the narrow distribution of states [2,24].

Coherent states of ion motion can be produced from the $|n=0\rangle$ state by a spatially uniform classical driving field [25], by a "moving standing wave" [26], by pairs of standing waves [8], or by a sudden shift of the trap center [5]. We have used the first two methods; for the data shown here we use the first. For the classical drive, we apply a sinusoidally varying potential at the trap oscillation frequency on one of the trap compensation electrodes [21] for a fixed time (typically $10 \mu \mathrm{s}$ ). In Fig. 3 we present an example of $P_{l}(t)$ after creation of a coherent state of motion, exhibiting collapse and revival. The time-domain data are fitted by Eq. (2) using a Poissonian distribution and allowing only $\bar{n}$ to vary. All other parameters are measured from a separate trace similar to Fig. 1(a). The inset shows the probabilities of the Fock components, extracted using the frequency-domain analysis described above. These amplitudes display the expected Poissonian dependence on $n$. The observed revival for higher $-\bar{n}$
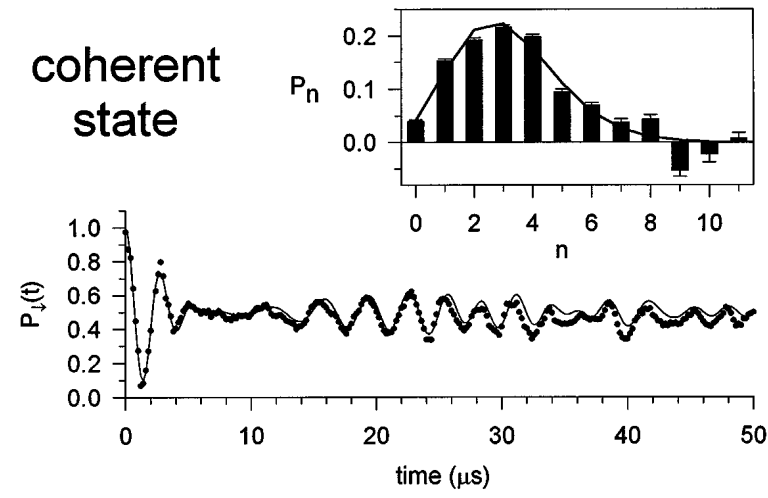

FIG. 3. $P_{l}(t)$ for a coherent state, showing collapse and revival. The data are fitted by a coherent state distribution, yielding $\bar{n}=3.1 \pm 0.1$. The inset shows the decomposition of the data onto the expected Fock state components, fitted by a Poissonian distribution, yielding $\bar{n}=2.9 \pm 0.1$.

coherent states is attenuated due to the progressively faster decay rates of the higher- $n$ Fock states, and for states with $\bar{n} \gtrsim 6$ we are unable to see the revival.

A coherent state has a definite phase relationship between the Fock state components. The signal $P_{\downarrow}(t)$, however, does not contain this phase information. To demonstrate the phase coherence of the created states, we apply a second pulse of classical driving force, which coherently returns the ion to the $|n=0\rangle$ state, provided the pulse is of the same amplitude as the first and $180^{\circ}$ out of phase with the coherent state. As expected, the return of the ion to the $|n=0\rangle$ state is very sensitive to the phase of the second pulse. However, we are able to reverse the coherent state and return the ion to $|n=0\rangle$ more than $85 \%$ of the time, as indicated by a single frequency component in a subsequent measurement of $P_{\downarrow}(t)$.

A "vacuum squeezed state" of motion can be created by a parametric drive [5], by a combination of standing- and traveling-wave laser fields [8], or by a nonadiabatic drop in the trap spring constant [5]. Here we irradiate the $|n=0\rangle$ ion with two Raman beams which differ in frequency by $2 \omega$, driving Raman transitions between the even- $n$ levels within the same hyperfine state. The interaction can also be thought of as a parametric drive induced by an optical dipole force modulated at $2 \omega$ [26]. The squeeze parameter $\beta$ (defined as the factor by which the variance of the squeezed quadrature is decreased) grows exponentially with the driving time. Figure 4 shows $P_{\downarrow}(t)$ for a squeezed state prepared in this way. The data are fitted by a vacuum squeezed state distribution, allowing only $\beta$ to vary. The fit of the data in Fig. 4 demonstrates consistency with a squeezed state and finds $\beta=40 \pm 10$, which corresponds to $\bar{n} \approx 7.1$.

The population distribution for a vacuum squeezed state is relatively flat and is restricted to the even states, $P_{2 n}=N(2 n) !(\tanh r)^{2 n} /\left(2^{n} n !\right)^{2}$, with $\beta=\exp (2 r)$. A squeezed state with $\beta=40$ has $16 \%$ of the population in states above $n=20$. The Rabi frequency differences of 


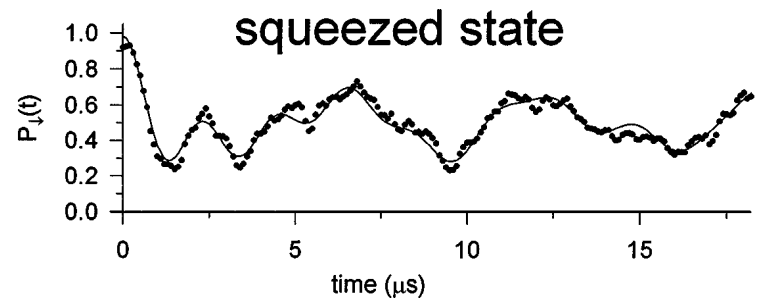

FIG. 4. $\quad P_{\downarrow}(t)$ for a squeezed state. The data are fitted by a squeezed state population distribution, finding $\beta \approx 40 \pm 10$, which corresponds to $\bar{n} \approx 7.1$.

these high- $n$ levels are small [see Fig. 1(b)], and with the inclusion of nonlinear effects $[19,20]$ the Rabi frequencies begin to decrease with $n$ after $n=20$. The levels can no longer be distinguished, and the frequency-domain analysis cannot be used to extract the level populations.

In summary, we have created thermal, Fock, coherent, and squeezed states of motion of a trapped ion and examined these states through the evolution of the ion internal state $P_{\downarrow}(t)$ induced by a (nonlinear) JCM-type interaction. This experiment demonstrates the utility of a trapped ion for the creation of nonclassical states of motion and investigations of the dynamics of Jaynes-Cummings-type interactions. Given a suitable coupling, it should be possible to transfer these nonclassical state properties to other harmonic oscillators including macroscopic oscillators [5]. In addition to work to reduce the decoherence, further efforts will involve the creation of arbitrary quantum states of motion, including macroscopic superposition states (Schrödinger's cat states) [3], investigation of the "two-phonon" Jaynes-Cummings model [27,28], quantum state endoscopy [29], and quantum state tomography [30]. The same interaction can be extended to prepare correlated internal states of two or more trapped ions for sensitive detection [7,13] or quantum computation [17].

This work is supported by the U.S. Office of Naval Research and the U.S. Army Research Office. We acknowledge important contributions by J. C. Bergquist and helpful comments on the manuscript by M. Stephens, C. S. Wood, and M. Young.

[1] E. T. Jaynes and F. W. Cummings, Proc. IEEE 51, 89 (1963).

[2] B. W. Shore and P. L. Knight, J. Mod. Opt. 40, 1195 (1993).

[3] Cavity Quantum Electrodynamics, edited by P. R. Berman (Academic Press, Boston, MA, 1994).

[4] J. N. Hollenhorst, Phys. Rev. D 19, 1669 (1979).

[5] D. J. Heinzen and D. J. Wineland, Phys. Rev. A 42, 2977 (1990).

[6] C. A. Blockley, D. F. Walls, and H. Risken, Europhys.
Lett. 17, 509 (1992).

[7] D. J. Wineland, J. J. Bollinger, W. M. Itano, F. L. Moore, and D. J. Heinzen, Phys. Rev. A 46, R6797 (1992).

[8] J. I. Cirac, A.S. Parkins, R. Blatt, and P. Zoller, Phys. Rev. Lett. 70, 556 (1993).

[9] J. I. Cirac, R. Blatt, A. S. Parkins, and P. Zoller, Phys. Rev. Lett. 70, 762 (1993).

[10] H. Zeng and F. Lin, Phys. Rev. A 48, 2393 (1993).

[11] J. I. Cirac, R. Blatt, A. S. Parkins, and P. Zoller, Phys. Rev. A 49, 1202 (1994).

[12] J. I. Cirac, R. Blatt, and P. Zoller, Phys. Rev. A 49, R3174 (1994).

[13] D. J. Wineland, J. J. Bollinger, W. M. Itano, and D. J. Heinzen, Phys. Rev. A 50, 67 (1994).

[14] R. Blatt, J. I. Cirac, and P. Zoller, Phys. Rev. A 52, 518 (1995).

[15] H. Zeng and F. Lin, Phys. Rev. A 52, 809 (1995).

[16] J. Eschner, B. Appasamy, and P.E. Toschek, Phys. Rev. Lett. 74, 2435 (1995).

[17] J. I. Cirac and P. Zoller, Phys. Rev. Lett. 74, 4091 (1995); C. Monroe, D. M. Meekhof, B. E. King, W. Itano, and D. J. Wineland, Phys. Rev. Lett. 75, 4714 (1995).

[18] C. Monroe, D. M. Meekhof, B.E. King, S. R. Jefferts, W. M. Itano, D. J. Wineland, and P. L. Gould, Phys. Rev. Lett. 75, 4011 (1995).

[19] See Eq. (31) in D. J. Wineland and W. M. Itano, Phys. Rev. A 20, 1521 (1979).

[20] W. Vogel and R. L. de Matos Filho, Phys. Rev. A 52, 4214 (1995).

[21] S. Jefferts, C. Monroe, E. Bell, and D. J. Wineland, Phys. Rev. A 51, 3112 (1995).

[22] S. Stenholm, Rev. Mod. Phys. 58, 699 (1986).

[23] W. H. Press, S. A. Teukolsky, W. T. Vetterling, and B. P. Flannery, Numerical Recipes (Cambridge University Press, Cambridge, 1992).

[24] J.H. Eberly, N. B. Narozhny, and J. J. SanchezMondragon, Phys. Rev. Lett. 44, 1323 (1980).

[25] P. Carruthers and M. M. Nieto, Am. J. Phys. 7, 537 (1965).

[26] D. J. Wineland, J.C. Bergquist, J.J. Bollinger, W. M. Itano, F. L. Moore, J. M. Gilligan, M. G. Raizen, D. J. Heinzen, C.S. Weimer, and C.H. Manney, in Laser Manipulation of Atoms and Ions, edited by E. Arimondo, W.D. Phillips, and F. Strumia (North-Holland, Amsterdam, 1992), p. 553.

[27] P. L. Knight, Phys. Scr. T12, 51 (1986).

[28] R. L. de Matos Filho and W. Vogel, Phys. Rev. A 50, R1988 (1994).

[29] P. J. Bardroff, E. Mayr, and W. P. Schleich, Phys. Rev. A 51, 4963 (1995), and references therein.

[30] K. Vogel and H. Risken, Phys. Rev. A 40, 2847 (1989); D. T. Smithey, M. Beck, M. G. Raymer, and A. Faridani, Phys. Rev. Lett. 70, 1244 (1993); T. J. Dunn, I. A. Walmsley, and S. Mukamel, Phys. Rev. Lett. 74, 884 (1995); S. Wallentowitz and W. Vogel, Phys. Rev. Lett. 75, 2932 (1995); J.F. Poyatos, R. Walser, J. I. Cirac, P. Zoller, and R. Blatt (to be published). 